\title{
Vista Dinámica y Estática de la Coordinación de Procesos de Negocios: Una Aproximación basada en Restricciones
}

Jorge E. Giraldo ${ }^{1,3}$, Sandra P. Mateus ${ }^{2}$ y Maryem A. Ruiz ${ }^{3}$

(1) Universidad Nacional de Colombia, Facultad de Minas, Núcleo Robledo, Escuela de Sistemas, Cr 80 \# 2-23, Medellín, Antioquia-Colombia (e-mail: jegiraldp@unal.edu.co)

(2) Institución Universitaria de Envigado, Facultad de Ingeniería, Cr 29B \# 39 A Sur 57, Envigado, Antioquia-Colombia (e-mail: spmateus@iue.edu.co)

(3) Politécnico Colombiano Jaime Isaza Cadavid, Sede Poblado, Facultad de Ingenierías,

Cr 48 \# 7 - 151 Medellín, Antioquia-Colombia (email: [jegiraldo, mruiz]@elpoli.edu.co)

\section{Resumen}

Este artículo presenta una aproximación al modelado de la coreografía de servicios Web basado en restricciones para lograr cierto grado de automatización en su definición. Se plantea la definición tanto desde el punto de vista estructural como dinámico de los componentes de la coreografía de servicios Web. Esto con el fin de realizar su representación en términos de un problema de verificación de restricciones. Se presentan los resultados de rendimiento del proceso comparado con otras aproximaciones, demostrándose que el modelo es apropiado para lograr automatización en la descripción del proceso de coreografía de servicios Web.

Palabras clave: procesos de negocios, servicios web, integración de servicios, coreografía web

\section{Static and Dynamic View of the Business Process Coordination: An Approach based on Constraints}

\begin{abstract}
This article presents an approach for modelling the choreography of the Web services based on constraints in order to achieve a certain degree of automation in its definition. The definition from both the structural and component dynamics of the Web services choreography. This with the objective of doing its representation in terms of a problem of restriction verification. Results of process performance are compared with other approaches, showing that the model is appropriate to achieve automation in the description of the process choreography Web services.
\end{abstract}

Keywords: business process, web services, service integration, web choreography 


\section{INTRODUCCIÓN}

El desarrollo de software orientado a componentes y en especial de una aplicación web se caracteriza por la tendencia de generar paquetes modulares de software, independientes de la plataforma demonizados Servicios Web - SW -. Su aporte principal es proveer funcionalidades de negocio a otras aplicaciones a través de internet (Srivastava y Koehler, 2003), adicionalmente permitir el desarrollo de tecnologías e investigaciones que los hacen propios para soluciones en dominios de tipo distribuido.

Bajo el enfoque web, los Servicios desempeñan distintos roles dentro del proceso de la lógica de un negocio (SOC) (Papazoglou, 2003), la interacción y envío de información a través de estos se conoce como Coordinación de Servicios. Últimamente se emplean dos conceptos complementarios entre si que permiten diseñar una Coordinación de Servicios Web, estos son la Coreografía y Orquestación (Peltz, 2003), siendo el primero de estos interés de este artículo.

En un proceso de Coreografía se intercambia información entre los servicios y sus operaciones,; para lograr cierto grado de automatización del proceso Coordinación, esta información requiere ser controlada, lo cual se puede hacer mediante Técnicas como la Planificación en Inteligencia Artificial (Peer, 2005), Técnicas de Búsquedas Heurísticas y en especial la Verificación de Restricciones (Rich y Knight, 1994). Se pretende entonces modelar la Coreografía de Servicios Web con base en Restricciones definidas sobre su información con el fin de lograr cierto grado de automatización en esta. Este trabajo presenta el resultado de un modelamiento de coordinación de Servicios Web basado en restricciones.

\section{Coreografía y Orquestación de Servicios Web}

La Coreografía de SW se encarga de identificar la secuencia válida de mensajes entre servicios o procesos de negocios que interactúan entre si. Básicamente la Coreografía con respecto a la composición de SW es un mecanismo de diseño que pretende definir un comportamiento global de ésta a partir de comportamientos individuales que se relacionan por medio del intercambio de información y que se rigen por reglas de comportamiento (Peltz, 2003). Este flujo de información en algunos casos se denomina Interacciones o Conversaciones que ya se han sido tratados por otros autores (Hull et al., 2003). La Orquestación que persigue la definición del proceso de Composición desde una perspectiva individual y no es de su interés el comportamiento de los demás participantes (Chen et al., 2006).

Estos dos conceptos, Coreografía y Orquestación con frecuencia son confundidos debido a que ambos son participes del proceso de Composición de SW. La Orquestación enfatiza en la descripción del proceso de composición desde un punto de vista individual, invocando mensajes y enviándolos, aprovechando la interfaz que provee la descripción del Servicio. Por su parte la Coreografía presenta la definición de una colaboración entre Servicios para alcanzar un objetivo en común, basada en el intercambio público de mensajes que ocurre entre estos.

\section{Problemas de Verificación de Restricciones}

Un gran número de problemas en inteligencia artificial y otras áreas de la ciencia de la computación pueden ser modelados como Problemas de Verificación de Restricciones -PVR-. Un PVR sobre un conjunto finito de dominios, puede ser expresado de la siguiente manera (Russell y Norvig , 2004): Dado un conjunto de variables junto con los valores que pueden ser asignados a las variables y una lista de restricciones, se deben encontrar los valores para las variables que satisfagan las restricciones definidas.

Este trabajo propone la problemática referida a la automatización de la definición del proceso de Coreografía de Servicios., ya que en la composición la definición de componentes y de sus interacciones se hace por separado y de manera manual, generando perdida del control al incrementarse el número de servicios en los procesos de negocios. 


\section{ANTECEDENTES}

Los problemas de la coreografía han sido abordados por varios autores (McKinley et al., 1999; Bergstra et al., 2001; Benatallah et al., 2002; Burdett y Kavantzas, 2003; Foster et al., 2004; Brogi y Canal, 2004). Estas propuestas se caracterizan por presentar una visión monolítica de la Coreografía en donde la poca expresividad en la información que la comprende impide que sea posible su manipulación automática. A esto se suma que no se tiene en cuenta desde el punto vista sistémico tanto la información como el orden en que ésta es transmitida a lo largo del proceso. Una de las primeras aproximaciones bajo un ambiente de la Planificación en IA hechas al manejo de las restricciones en el ámbito de los SW es la propuesta hecha por (Aiello y Papazoglou, 2002). Posteriormente en (Lazovik et al., 2006) con base en la solución anterior, las restricciones son empleadas para la representación del problema planteado por el usuario, mediante la representación de estados de transición es posible la definición de los estados finales que de una forma u otra representan los objetivos a cumplir. En Channa et al. (2005), se propone la aplicación de la Verificación de Restricciones al problema de la Coreografía Automática de SW. En este trabajo las restricciones son definidas mediante una Ontología de dominio, representadas en clases, atributos, instancias y relaciones, entre otras. METEOR-S (Aggarwal et al., 2004) es una plataforma para la composición de SW dirigida por restricciones, la idea es seleccionar los SW a partir de sus propiedades, se compone de un analizador de restricciones; las restricciones se definen sobre los aspectos funcionales de los SW, al igual que propuestas anteriores se tiene en cuenta la QoS; en la calidad del servicio se tiene en cuenta el Costo de ejecución, Tiempo de ejecución de una operación, Confiabilidad, disponibilidad y Métricas específicas del dominio

\section{METODOLOGÍA PROPUESTA}

Como primera medida se identifican y se modelan los componentes de la Coreografía (Servicios, operaciones, Roles y mensajes); con base en esta información se hace una representación en términos de un Problema de Verificación de Restricciones y luego se emplean mecanismos de de programación para su manipulación automática.

\section{Componentes}

La Coreografía de SW se caracteriza por los siguientes componentes: Servicios y los mensajes intercambiados entre estos, los Servicios representan funciones de negocio, los mensajes son información intercambiada por servicios. Los conceptos y componentes aquí presentados se enfocan en la definición de la Coreografía desde un punto de vista estructural y dinámico, la primera se encarga de la definición de los componentes y sus características, la parte dinámica garantiza un apropiado intercambio de información durante la coordinación, es decir garantiza un flujo de información representado por medio de mensajes. En la siguiente figura se expone un modelado de la Coreografía de Servicios, los recuadros identifican cada una de las perspectivas de la Coreografía, tanto estructural como dinámica.

La información expuesta en la Figura 1 representa un diagrama de clases UML que expone la relación de los componentes que permiten la descripción de la Coreografía. Se ilustra que un Servicio Web está asociado con un solo Rol. Por su parte un Rol está definido a partir de Restricciones sobre la información que este brinda. Por último un servicio puede tener una o muchas operaciones asociadas a este y estas son incluidas en mensajes como parte de la información que este acarrea.

\section{Coreografía de Servicios Web como un Problema de Verificación de Restricciones}

Los siguientes elementos hacen posible ver la Coreografía de Servicios Web como un Problema de Verificación de Restricciones; estos son: las Variables, un Dominio para cada valor y un conjunto de Restricciones.

Variables: Identificador del Rol, Numero de Variables del Rol, Numero de Variables de entrada, número de Variables de salida y tipo de operación. 


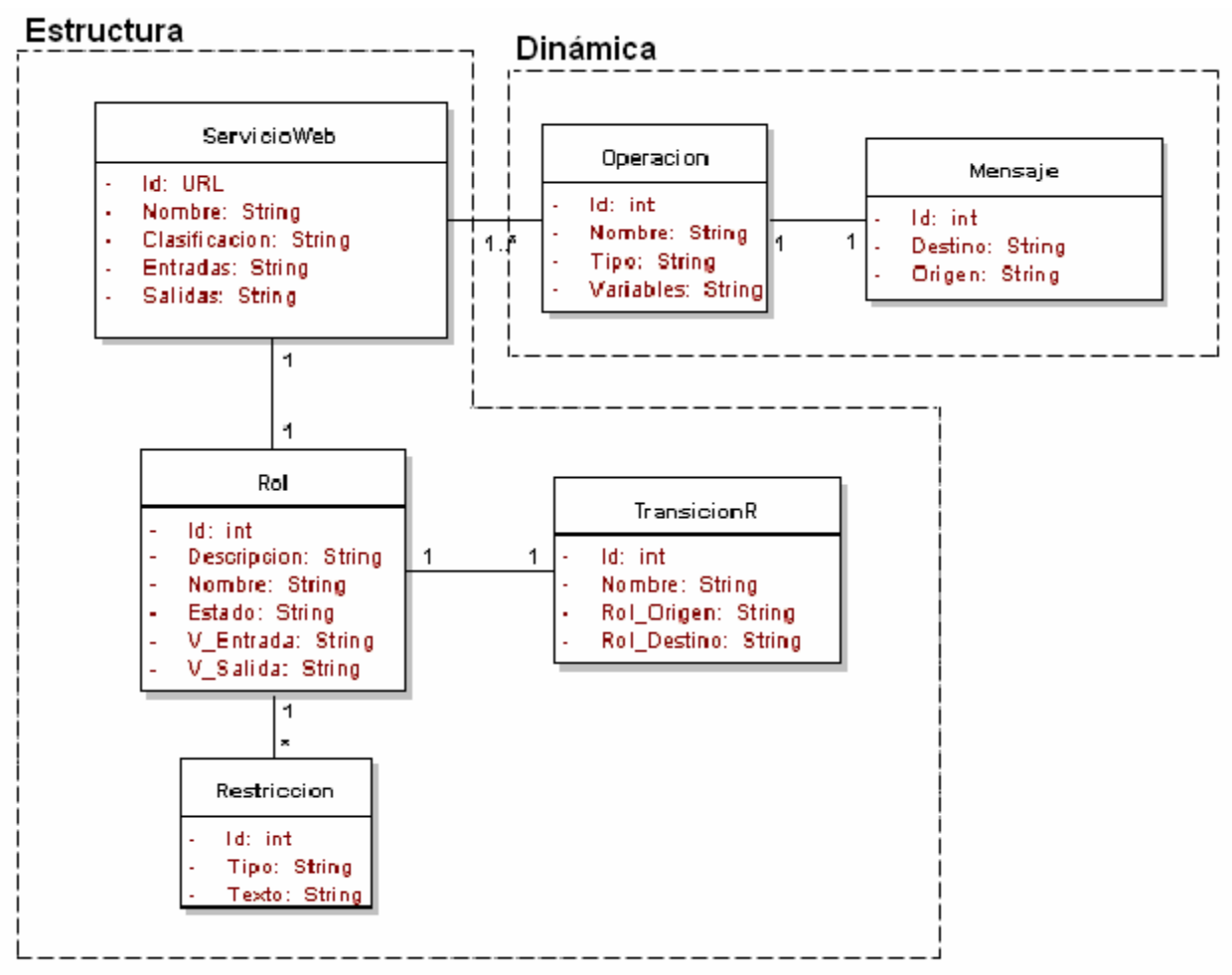

Fig. 1: Modelado Planteado

Dominios de los valores: Por cada una de las variables se debe determinar un valor para estas, los valores están regidos por un solo dominio y este es el repositorio de descripciones de servicios.

Restricciones: Son expresiones que condicionan la asignación de un valor a una variable, este valor pertenece a un dominio específico. Se busca entonces definir restricciones que busquen equivalencia de información entre la que otorgan el Proceso de Negocio y las descripciones de los Servicios Web.

Las Restricciones que se definen son: Restricciones de Tipo de Operación, de Número de Variables, de Variables de Entrada, de Salida y de Existencia del Servicio.

La primera restricción expresa que el Tipo de Operación propuesto por el Proceso de Negocio debe ser el mismo tipo que manifiesta una Operación de un Servicio. La segunda define que el total de variables manejadas por un Rol Proceso sean equivalentes del servicio analizado. La tercera y cuarta Restricción se definen sobre la información de las Variables de entrada y de salida respectivamente. Por último se encuentra la Restricción donde se plantea que para todos los Roles debe existir un valor asociado, esto garantiza que se asocie un Servicio a un Rol.

\section{Resultado Obtenidos, Comparación con otros Modelos}

La validación realizada al modelo planteado se basó en algunos aspectos orientados a medir el rendimiento del proceso de Definición Coreografía de Servicios, estos son: Escalabilidad, Complejidad en el proceso, Número de asignaciones, Falsos positivos, Falsos Negativos.

Las propuestas con las que se comparó el modelo son (Aggarwal et al., 2004), donde se presenta una plataforma para la composición de SW dirigida por restricciones que están definidas sobre los aspectos no funcionales de los SW, tales como: Costo de ejecución, Tiempo de Ejecución del proceso, Falsos positivos y Falsos Negativos. En Thakkar et al. (2005) se presenta un marco conceptual para el manejo de la composición, optimización y ejecución de planes para Servicios 
Web. Para validarla se mide el Tiempo de Composición dependiente del número de Servicios involucrados, criterio a utilizar para comparar el modelo propuesto. En la primera propuesta se tomaron los resultados con base en el caso de estudio asociado al dominio del turismo [Peer, 2003], sin embargo en Thakkar et al. (2005) se presentan resultados si asociar a ningún dominio en especifico. En la tabla 1 se exponen las medias con base en un número de seis servicios y desde el punto de vista de restricciones se emplearon un total de 12 para cada uno de los casos.

Tabla 1: Comparación Rendimiento

\begin{tabular}{|c|c|c|c|}
\hline Criterio & Modelo Propuesto & Aggarwal & Thakkar \\
\hline Complejidad & $1110 \mathrm{mls}$ & $1030 \mathrm{mls}$ & -- \\
\hline Falsos Positivos & 1 & -- & 2 \\
\hline Falsos Negativos & 0 & -- & 0 \\
\hline
\end{tabular}

\section{CONCLUSIONES}

La conclusión principal de este trabajo de investigación es que el Modelo de Verificación de Restricciones, ofrece mejores prestaciones para que la definición de un proceso de Coreografía se pueda automatizar.

De las mediciones hechas se puede demostrar que la complejidad demora un poco mas de tiempo con respecto a otros modelos, no obstante su precisión mejora, en referencia a los Falsos Negativos y Falsos positivos.

EL intercambio de mensajes es clave para el modelamiento de la coordinación de sw, por lo cual la Coreografía de Servicios Web debe ser descrita desde un punto de vista tanto estructural como dinámico. La Vista Estructural debe encargarse de la definición de los Servicios y sus operaciones, por su parte la Vista Dinámica, permite el control del flujo de la Información por medio del intercambio de mensajes y el uso de los Estados de Roles.

Como trabajos futuros en el área se debe profundizar en los mecanismos de interacción y su interoperatividad semántica. Para ello es necesario buscar aplicaciones donde sea posible intercambiar información de manera masiva y así se evalúe la calidad de la información intercambiada.

\section{REFERENCIAS}

Aggarwal, R., K. Verma, J. Miller y W. Milnor; Constraint Driven Web Service Composition in METEORS. Services Computing, 2004 IEEE International Conference on (SCC'04) (2004).

Aiello, M. y M. Papazoglou; A Request Language for Web-Services Based on Planning and Constraint Satisfaction. Workshop on Technologies for E-Services (TES02) (2002).

Benatallah, B., M. Dumas, Q.Z. Sheng y A. Ngu; "Declarative Composition and Peer-to-Peer Provisioning of Dynamic Web Services". International IEEE Conference on Data Engineering, San Jose, USA (2002).

Bergstra, J., A. Ponse y A. Smolka; Editors. Handbook of Process Álgebra. Elsevier (2001).

Brogui, A. y C. Canal; "Formalizing Web Service Coreographies". Notes in Theoretical Computer Science: 105, 73-94 (2004).

Burdett, D. y N. Kavantzas; "Net Traveler: A Framework for Automatic Web Services Collaboration, Orchestation and Choreography in E-Government Information System". Universidad de Puerto Rico, (2003). 
Channa, N., L. Camping, A. Wasim y F. Xiangjun; Constraint Satisfaction in Dynamic Web Services Composition. Asian Journal of Information Technology: 4 (10), 957-961 (2005)

Chen, L., B. Wassermann, W. Emmerich y H. Foster; Service Orchestration with BPEL, IEEE International Conference on Software Engineering (ICSE 2006), Shanghai, China, May (2006).

Foster, H., S. Uchitel y J. Magee; Compatibility Verificaction for Web Services Choreography. IEEE ICWS 2004, San Diego, CA, (2004).

Hull, R., M. Benedikt, D. Christophides y J. Su; E-services: A look behind the curtain. In Proceedings of ACM Symposium on Principles of Database Systems. ACM (2003).

Lazovik, A., M. Aiello y R. Gennari; Choreographies: using Constraints to Satisfy Service Requests. In IEEE Web Services-based Systems and Applications (WEBSA at ICIW), (2006).

McKinley, P., A. Malenfant y J. Arango; Pavilion: a middleware framework for collaborative Web-based applications. Proceedings of the international ACM SIGGROUP conference on Supporting group work, November (1999).

Papazoglou, M.; Service oriented computing: Concepts, Characteristics and Directions. Tilburg University, INFOLAB. Dept. of Information System and Manegement (2003).

Peer, J.; Web services Composition as Al planning, A Survey. Second revised version, March 22 (2005).

Peltz, C. Web Services Orchestration and Choreography. Hewlett-Packard Company. IEEE Computer Society (2003).

Rich, E. y K. Knight; Inteligencia artificial. Segunda edición. McGrawHill (1994).

Russell, R. y P. Norvig; Inteligencia Artificial. Un enfoque Moderno. Segunda Edición. Pearson Education, S.A, Madrid (2004).

Srivastava, B. y J. Koehler;. Web Services Composition - Current Solutions and Open Problems. IBM research laboratory, pag. 28-35 (2003)

Thakkar, S., J. Ambite y C. Knoblock; Composing, optimizing, and executing plans for bioinformatics web services. The VLDB Journal:14(3), 330-353 (2005). 